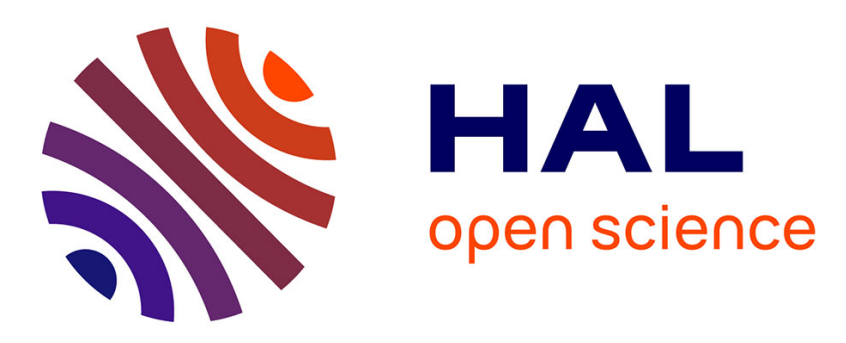

\title{
Two-Dimensional Hybrid Simulations of a Magnetosheath Filament Interaction with the Magnetopause
}

Ph. Savoini, M. Scholer

\section{- To cite this version:}

Ph. Savoini, M. Scholer. Two-Dimensional Hybrid Simulations of a Magnetosheath Filament Interaction with the Magnetopause. Journal de Physique IV Proceedings, 1995, 05 (C6), pp.C6-25-C6-30. 10.1051/jp4:1995605 . jpa-00253968

\section{HAL Id: jpa-00253968 https://hal.science/jpa-00253968}

Submitted on 1 Jan 1995

HAL is a multi-disciplinary open access archive for the deposit and dissemination of scientific research documents, whether they are published or not. The documents may come from teaching and research institutions in France or abroad, or from public or private research centers.
L'archive ouverte pluridisciplinaire HAL, est destinée au dépôt et à la diffusion de documents scientifiques de niveau recherche, publiés ou non, émanant des établissements d'enseignement et de recherche français ou étrangers, des laboratoires publics ou privés. 


\title{
Two-Dimensional Hybrid Simulations of a Magnetosheath Filament Interaction with the Magnetopause
}

\author{
Ph. Savoini and M. Scholer* \\ CETP/CNRS, 38-40, rue du Général Leclerc, 92131 Issy-les-Moulineaux, France \\ * Max-Planck-Institut für Extraterrestrische Physik, Garching, Germany
}

\begin{abstract}
Two-dimensional hybrid simulations are used to investigate the impulsive penetration process when a magnetosheath plasma irregularity interacts with the magnetopause. The irregularity is modeled as a field-aligned filament with an excess density impinging on the magnetopause. Different configurations are used defined by a parallel or anti-parallel magnetic field in both regions (magnetosheath and magnetosphere) and also in the case of sheared magnetic fields. The results show that a MHD approach is not totally usefull and emphasize the kinetic effects of the ions on the propagation of the plasma irregularities and their consequences on the energy balance between the two regions. Moreover, sheared magnetic field configurations allow inferences about the actual solar wind - magnetosphere interaction and show that the threshold conditions for penetration are much more restrictive than MHD results. This study strongly harms the impulsive penetration mechanism to explain the transfer of mass and energy from the magnetosheath to the magnetopshere.
\end{abstract}

\section{INTRODUCTION}

One of the major questions in magnetospheric research is how mass, momentum, and energy is transferred from the solar wind into the magnetosphere. Both indirect and in - situ observations [1,2] indicate that magnetic reconnection, first proposed by Dungey [3], is the dominant process. Viscous interaction between the solar wind and the magnetosphere due to the turbulence in the solar wind, proposed by Axford and Hines [5], seems to be of minor importance and acts mainly during times of northward interplanetary magnetic field. Furthermore, the solar wind is usually non-uniform over distances much smaller than the diameter of the magnetosphere and changes rapidly in time [4]. Measurements of plasma filaments within the magnetosphere may provide some evidence that the solar wind filaments have penetrated into the magnetosphere across closed magnetic field lines [5, 6]. Two theories [7, 8], close but distinct have been proposed to explain how a cloud of magnetosheath plasma having "excess" momentum penetrates into the magnetosphere. Two-dimensional MHD simulations [9] have been performed to consider the threshold conditions under which penetration might occur at the dayside magnetopause. They showed that the impulsive penetration mechanism is unlikely as a real important process at the magnetopause unless the $\mathbf{B}$ shear in within $5^{\circ}$. Nevertheless, such simulations have to be extended to a more realistic case where inertial effects and particle diffusion are selfconsistently included [10]. It is the goal of the present paper to investigate the efficiency of the impulsive penetration process in more realistic conditions that employ electromagnetic effects and ion kinetics.

\section{SIMULATION MODEL}

The present simulations have been performed with a two-dimensional electromagnetic hybrid code in which the ions are treated as macro-particles and the electrons as a charge neutralizing massless fluid. This means that physics involving electron mass effects is absent whereas the ions can exhibit the full range of kinetic phenomena. All quantities are normalized in the following way: the dimensionless variables $\tilde{x}, \tilde{y}$ (space coordinates), the time $\tilde{t}$, and the ion velocity $\tilde{v}=\tilde{v}_{x} ; \widetilde{v}_{y}, \widetilde{v}_{z}$, by the ion inertial length $\tilde{\lambda}_{2 \mathrm{i}}$, the inverse of the ion Larmor frequency $\tilde{\omega}_{2 \mathrm{ci}^{-1}}$, and by the Alfvèn velocity $\tilde{v}_{2 \mathrm{~A}}$ respectively, all taken within the magnetosphere (the subscripts 1,2 refers to the magnetosheath and the magnetospheric values, respectively). The time step is $\Delta \mathrm{t}=0.02$ and the numerical grid spacing is $\Delta \mathrm{x}=\Delta \mathrm{y}=0.5$. 
Furthermore, the extent of the simulation box is $\mathrm{L}_{x}=200 \lambda_{i}$ (normal to the boundary layer) and $\mathrm{L}_{\mathrm{y}}=50$ $\lambda_{\mathrm{i}}$ (along the boundary layer). Periodic boundary conditions for the particles and fields are employed in the y-direction along the magnetopause (justified because the diameter of the plasmoid is small compared to $\mathrm{L}_{\mathrm{y}}$ ). The Earth's magnetopause is modeled as a tangential discontinuity with no magnetic field component and no mass flow normal to the surface. All parameters across the magnetopause are defined by a hyperbolic tangent function. We assume in the simulation runs a magnetopause thickness of $3 \lambda_{\mathrm{i}}(\sim 500 \mathrm{~km})$. The magnetosheath irregularity is modeled as a cylindrical filament with a diameter equal to three times the width of the magnetopause boundary layer, $\Delta_{\mathrm{F}}=9 \lambda_{\mathrm{i}}$ and an enhanced plasma density of $n_{F}=10 n_{2}$. Initially, the plasma filament has a bulk speed of $\widetilde{v}_{F}=0.7$ in the $x$ - direction.

\section{SIMULATION RESULTS}

The numerical experiments show that the nonideal MHD processes like particle inertia effect, anisotropic pressure, and the Hall term significantly modify the impulsive plasma penetration mechanism. Therefore, MHD considerations previously used to quantify this process [11] are probably not immediately applicable in the determination of penetration criteria. In the following, we will present separately results obtained for the anti- (used as a reference case), the parallel and a sheared case.

\subsection{Results for the anti-parallel case}

The anti-parallel case is initialized by assuming positive magnetic component in the magnetosheath and a negative one in the magnetosphere. This configuration is characterized by a very strong magnetic gradient at the transition. The anti-parallel case has the advantage that the localisation of the current sheet is rather evident (zero $B_{Z}$ component) which allows the definition of the magnetopause position in a first approximation (heavy line). Figure 1 (left panels) displays the time evolution of an elarged view of the magnetosheath density (plasmoid included).

As reference, we have included the initial state, $t=0$. Somewhat later in time, the plasma filament has intersected the current sheet and makes a dent in the magnetosphere surface. The other plots illustrate the subsequent deformation and motion of the plasmoid as it moves further into the magnetospheric region. The global plasmoid behavior can be divided into two differents phases: (i) the first phase is characterized by the intrusion of the plasmoid into the magnetosphere whereas it maintains its identity and continues to be always associated with a large flow speed in the $\mathrm{x}$-direction. As a results, the magnetopause is strongly deformed and draped around the plasmoid in a finger-like shape $(\mathrm{t} \leq 180)$, (ii) the second phase is mainly due to the action of the vortices onto the plasmoid. Indeed, the computations show that the "dense" plasma as it penetrates into the magnetosphere, sets up a circulatory flow, in which the "light" plasma (magnetospheric population) is deflected in front of and out to the sides. The rightward push causes draining along the side of the structure, resulting in the characteristic doublevortex pattern. Vortices wound and stretch the magnetopause boundary and induce a magnetic field cavity (Figure $1, t=160$ ), especially at the "port side" tail where the density is very low $\left(n_{p l}\right.$ is below 2 ). As a result, the deformation of the magnetopause becomes the well known mushroom-like shape obtained in fluid studies of a heavy fluid funneling downward into an underlying light fluid. The nonsymetric property of the anti-parallel configuration is an important result of these present kinetic simulations.

The presence of a differential rotation between the magnetosphere and the magnetosheath medium due to the inertia effect of the plasmoid ions leads to macroscopic instabilities such as the Kelvin-Helmholtz $(\mathrm{K}-\mathrm{H})$ instability [e.g., 12, 13]. The existence of such an instability is suggested from the magnetic field contours (in particular from the $\mathrm{B}=0$ line) where ripples sligthly deform the magnetopause which spontaneously diffuses when they are drawn sufficiently close together. A part of the plasmoid breaks into pieces (magnetosheath islands) surrounded by the part of the plasmoid population which has directly diffused througth the magnetopause.

It is found that magnetospheric particles which interact with the plasmoid structure (under the action of ExB drift) exhibit a strong heating. During a short period $\left(\Delta_{t} \approx 20\right)$, the thermal energy gain is about 24 times its initial main energy. An appreciable number of particles is strongly accelerated which leads to an increase of the total magnetosphere energy, $\mathrm{E}_{\text {tot }}{ }_{\text {final }} / \mathrm{E}_{\text {tot }}$ initial $\approx 1.8$. 


\subsection{Results for the parallel case}

In this configuration, all initial parameters are the same, while the magnetic fields are now parallel. In regard to the plasmoid penetration, the most important point connected with this simulation is the absence of a strong magnetic field gradient at the magnetopause boundary. Contrary to the previous case, the main part of the plasmoid is focused into a small region during all its penetration. Therefore, its main density is much higher than in the anti-parallel case (about twice at the end of the simulation, $t=304$ ) which increases its excess momentum density with respect to the background medium. As in the MHD simulations [11], the penetration depth is then bigger. The filament maintains its identity as it propagates in a straight line to the other side of the simulation domain, leaving two tails of enhanced density in the magnetosphere, see Figure 1, right panels.

\subsection{Results for a sheared case}

In this case, the magnetic field in the magnetopshere is oriented at an angle of $3.82^{\circ}$ to the magnetic field in the magnetosheath (corresponding to a $\mathrm{y}$-component $\mathrm{B}_{2 \mathrm{y}}=0.02$ ), allowing a comparison wiyh $\mathrm{MHD}$ results [11]. In opposite to the previous cases, the transverse component of magnetic field resists the penetration of the filament. The global behavior is totally different and is depicted in Figure 2 which shows in the same format than Figure 1 the magnetosheath density. As the filament goes farther into the magnetosphere, the magnetic flux increases in front of it and decelerate the blob of plasma. Figure 2 clearly shows that the plasmoid does not penetrate the magnetosphere. The evolution of the current density shows that no significant current sheet develops behind the filament at any time during this simulation. Consequently, reconnection is not expected, even if we had included a current-dependent enhancement to the local resistivity in the simulation model.

New features appear concerning the magnetospheric population. Particularly, the action of vortex structures combined with the increasing of the magnetic flux in front of the plasmoid leads to a repelling of the "port side" part of the plasmoid. The amplitude of the magnetosheath movement is strong enough to squeeze the magnetic field lines and induces spontaneaous reconnection. Figure 3 shows the time evolution of an elarged view of the $B$ lines in the simulation plane at the reconnection position. As expected, the plasma is accelerated on each side and a part of the magnetospheric population is projected into the magnetosheath region. This blob of plasma has sufficient kinetic energy to cross through the filament and to return to the magnetosphere. No acceleration is observed in the opposite direction due to the ExB vortex drift which confines the plasma.

\section{DISCUSSIONS AND CONCLUSIONS}

These simulations reveal the importance of the magnetospheric ions in the generation of the vortex structures and their dominant action on the propagation of the plasmoid. Under the ExB drift, particles of the magnetospheric region are swepted toward the plasmoid structure. The scale length of these convection twin vortices is about $40 \lambda_{i}$ and thus is much larger than the typical plasmoid scale length $\left(\Delta_{\mathrm{pl}}=9 \pi_{\mathrm{i}}\right)$. In this respect, it is interest to note that similar observations have been made concerning the ionospheric traveling convection vortices induced by the dynamic response of the Earth's magnetosphere to the pressure pulses at the magnetopause $[14,15]$. The resulting drift of the magnetospheric population allows a large number of particle to interact with the plasmoid structure, even those particles which are totally out of its way and without direct interaction with it. In this way, the induced motion in the magnetosphere is important for the transfer of energy and momentum from the plasmoid to the magnetospheric population.

The generalisation for more complicated configurations with a sheared magnetic field between the interplanetary magnetic field (IMF) and the Earth emphasizes the limitations of the MHD paradigm for this process. In order for penetration to occur, Ma et al. [11] define a impulsive penetration threshold R:

$$
R=\frac{1}{2} \rho_{F} v_{F}^{2} / \frac{B_{2 y}^{2}}{2 \mu_{o}} \geq 50
$$

where $\rho_{F}$ is the filament density, $v_{F}$ its initial velocity, which represents the ratio between the initial kinetic energy density of the filament and the $B_{y}$ magnetic energy density. They conclude that the impulsive penetration may occur unless the $\mathbf{B}$ shear is within $5^{\circ}$ and they argue that ideal MHD theory is too restrictive for such an impulsive penetration mechanism. 
In contrary, our kinetic simulations demonstrate the real difficulty for a blob of plasma to penetrate into the magnetosphere, even when we use a very high value for the penetration threshold, up to $\mathrm{R}=1160$ (to be compare to $\mathrm{R}=50$ of Ma et al. [11]).

Only for strictly anti- and parallel cases, an appreciable particle diffusion is observed which leads to a large increase of the magnetosphere energy, $\mathrm{E}_{\mathrm{mp}}$ final $/ \mathrm{E}_{\mathrm{mp}}$ initial $\approx 1.8$. This can be associated with the $\mathrm{K}$ $H$ instability which converts the energy of relative bulk plasma flow (in our case, the filament) into other forms (e. g. thermal energy).

At this point, we have to point out that the present paper can not to evaluate the real importance of the impulsive penetration model in the global scenario explaining the transfer of energy, mass and momentum from the magnetosheath (the shocked solar wind) into the magnetosphere. The line-typing of field lines in the ionosphere is also important in a global scenario. Furthermore, three dimensional simulations are necessary if the field-aligned dimension of the plasma irregularity is not small compared to the dimensions of the magnetosphere.

\section{References}

[1] Paschmann, G., B. U. Ö. Sonnerup, I. Papamastorakis, N. Sckopke, G. Haerendel, S. J. Bame, J. R. Asbridge, J. T. Gosling, C. T. Russelll and R. C. Elphic, Nature 2821979243.

[2] Sonnerup, B. U. Ö., G. Paschmann, I. Papamastorakis, N. Sckopke, G. Haerendel, S. J. Bame, J. R. Asbridge, J. T. Gosling and C. T. Russell, J. Geophys. Res. 86 (1981) 10049-10067.

[3] Dungey, J. W., Phys. Rev. Lett. 6 (1961) 47.

[4] Harvey, C. C., J. Etcheto, J., and A. Mangeney, Space Sci. Rev.23 (1979) 39.

[5] Sckopke N., G. Paschmann, G. Hearendel, Sonnerup, S. J. Bame, and E. W. Jr. Hones, J. Geophys. Res. 86, (1981) 2099-2110.

[6] Lundin, R., Planet. Space Sci. 32, (1984), 757.

[7] Axford, W. I., and C. O. Hines, Can. J. Phys. 39 (1961) 33.

[8]Lemaire, J., J. Plasma Physics 33, 425, 1985.

[9] Heikkila, W. J., Geophys. Res. Lett. 9, 159, 1982.

[10] Savoini, Ph., M. Scholer and M. Fujimoto, J. Geophys. Res 99, (1994) 19377.

[11] Ma, Z. W., J. G. Hawkins, L. C. Lee, J. Geophys. Res. 96, (1991) 15,751-15765.

[12] Belmont, G. and G. Chanteur, Phys. Scri. 40, (1989) 124.

[13] Pu, Z. Y., Y. Ming and Z. X. Liu, J. Geophys. Res. 95, (1990) 10559.

[14] Friis-Christensen, E., M. A. McHenry, C. R. Clauer, S. Vennerstrom, Geophys. Res. Lett. 15, (1988) 253.

[15] Glassmeier, K.H. and C. Heppner, J. Geophys. Res. 97, (1992) 3977. 

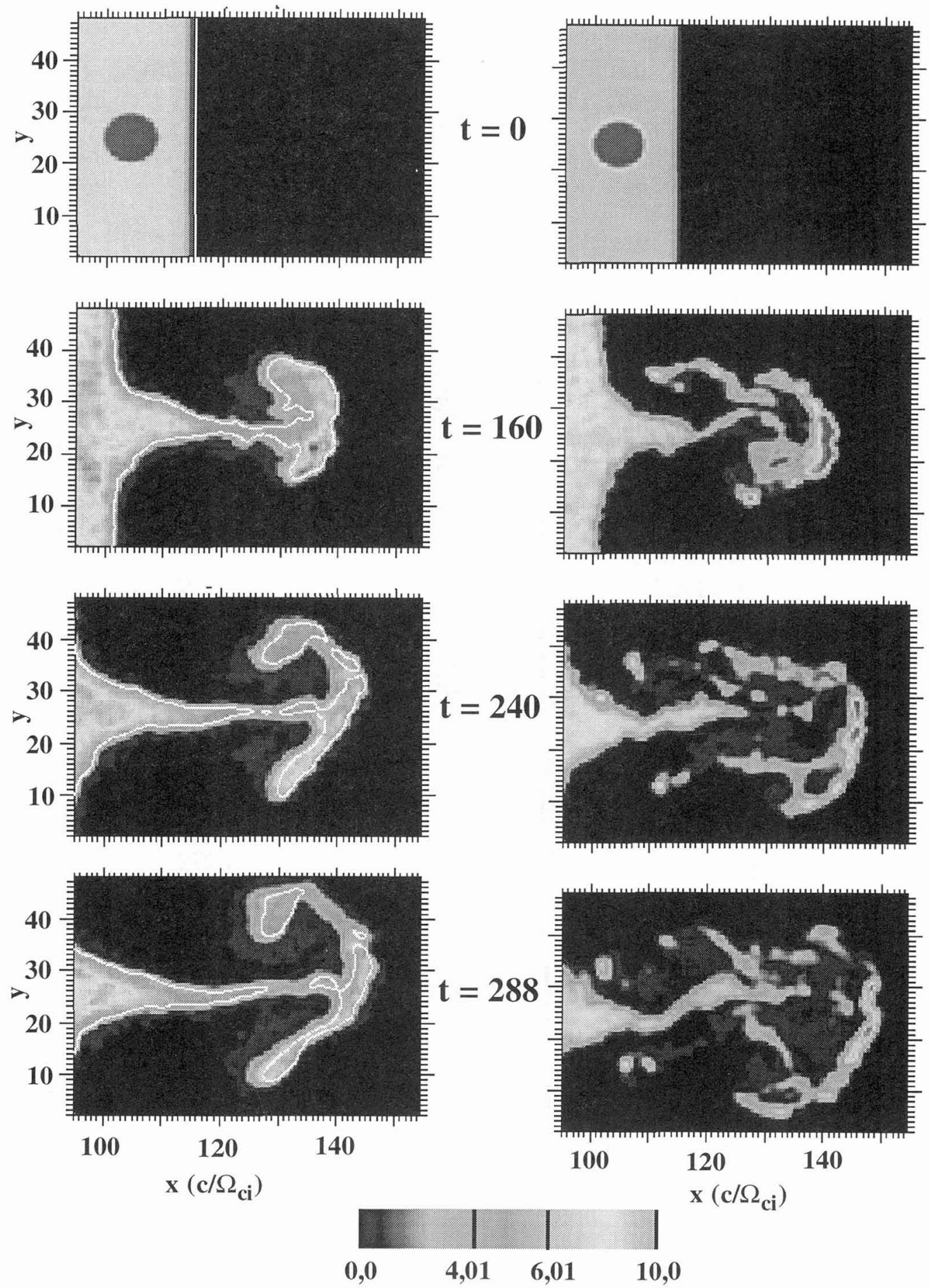

Figure 1: Ion density of the magnetosheath at $\Omega_{\mathrm{ci}} \mathrm{t}=0,160,240,288$ showing the penetration process and the deformation of the magnetopause for the antiparallel case (left panel) and the parallel case (right panel). For the antiparallel case, the magnetopause is reprensented by the heavy line $(\mathrm{B}=0)$. 

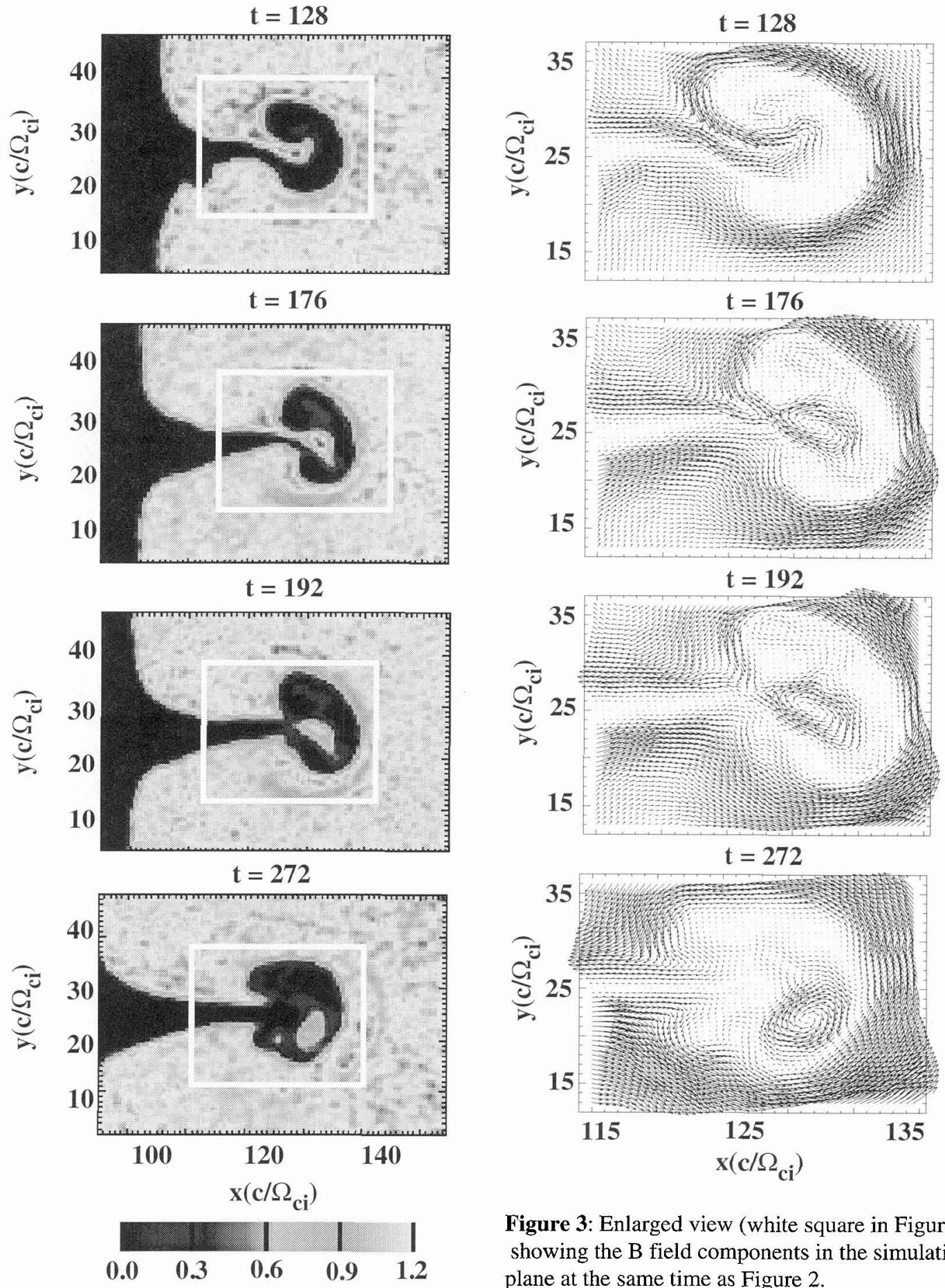

Figure 3: Enlarged view (white square in Figure 2) showing the $\mathrm{B}$ field components in the simulation plane at the same time as Figure 2.

Figure 2: Ion density of the magnetosphere at $\Omega_{\mathrm{ci}} \mathrm{t}=128,176,192,272$ for the shear case for $\mathrm{B}_{\mathrm{y}}=0.06$ 